\section{ADA EVENTS}

73rd Scientific Sessions

21-25 June 2013

McCormick Place Convention Center

Chicago, Illinois

Web site: http://scientificsessions.diabetes.org

Diabetes is Primary

22 June 2013

Chicago, Illinois

Contact: Shirley Ash

E-mail: sash@diabetes.org

Web site: professional.diabetes.org/primary

Endocrinology Seminar

19 September 2013

Crowne Plaza Hotel

Cromwell, Connecticut

E-mail: lbutcher@diabetes.org

6th Disparities Partnership Forum

21-22 October 2013

Hilton Crystal City

Arlington, Virginia

Contact: Debra Kilpatrick Byrd

E-mail: dkbyrd@diabetes.org

Web site: professional.diabetes.org/disparitiesforum

11th Professional Symposium on Diabetes

26 October 2013

The Queen's Conference Center in The Queen's Medical Center

Honolulu, Hawaii

E-mail: lduenas@diabetes.org

Web site: professional.diabetes.org/hawaii

Annual Diabetes Practice Update

1 November 2013

DoubleTree by Hilton Portland

Portland, Oregon

E-mail: jgoodrich@diabetes.org

Web site: professional.diabetes.org/oregon

22nd Annual Conference on Diabetes

1-2 November 2013

Seminole Hard Rock Resort

Fort Lauderdale, Florida

E-mail: bggalan@diabetes.org

Web site: professional.diabetes.org/sfl 39th Annual Diabetes Symposium

2 November 2013

Hyatt Regency Wichita

Wichita, Kansas

Contact: Patricia Tasker

E-mail: ptasker@diabetes.org

Web site: professional.diabetes.org/kansas

44th Annual Symposium on Diabetes for Healthcare Providers

6 November 2013

Crowne Plaza Hotel

Cromwell, Connecticut

Contact: Louise Butcher

E-mail: lbutcher@diabetes.org

Web site: professional.diabetes.org/ashcp

Stop Diabetes Awards of Excellence

22 November 2013

Cleveland, Ohio

Contact: Joann Mraz

E-mail: jmraz@diabetes.org

Web site: professional.diabetes.org/ohio

61st Annual Advanced Postgraduate Course

7-9 February 2014

Hyatt Regency San Francisco

San Francisco, California

Contact: Shirley Ash

E-mail: sash@diabetes.org

Web site: professional.diabetes.org/pg14

74th Scientific Sessions

13-17 June 2014

Moscone Center

San Francisco, California

Web site: http://scientificsessions.diabetes.org

75th Scientific Sessions

5-9 June 2015

Boston Convention and Exhibition Center

Boston, Massachusetts

Contact for information on ADA events: American Diabetes Association, 1701 N. Beauregard St., Alexandria, VA 22311.

Tel: 800-232-3472, select option 1. Fax: 703-549-1715

or 703-253-4358. E-mail: professionaleducation@diabetes.org.

Web site: $\mathrm{http} / / /$ professional.diabetes.org/ce.

\section{OTHER EVENTS}

81st Congress of the European Atherosclerosis Society 2-5 June 2013

Lyon, France

E-mail: eas@kenes.com

Web site: www.kenes.com/eas2013

Diabetes Innovation 2013

3-5 October 2013

Omni Shoreham Hotel

Washington, DC

E-mail: nancy.felsheim@joslin.harvard.edu

Web site: $h t t p: / / w w w . d i a b e t e s i n n o v a t i o n . o r g$ 8th World Congress on Developmental Origins of Health and Disease

17-20 November 2013

SUNTEC Singapore

Web site: http://www.dohad2013.org

7th International Conference on Advanced Technologies and Treatments for Diabetes

5-8 February 2014

Vienna, Austria

E-mail: attd@kenes.com

Web site: www.kenes.com/attd2014 